\title{
Research Progress of Heat Shock Protein 90 and Hepatocellular Carcinoma
}

\author{
Qiyu Jiang, Xiongshan Shen* \\ Department of General Surgery, Xiaogan Hospital Affiliated to Wuhan University of Science and Technology, Xiaogan, \\ China \\ Email: jiangtaiyu@foxmail.com, ^2440672138@qq.com
}

How to cite this paper: Jiang, Q.Y. and Shen, X.S. (2020) Research Progress of Heat Shock Protein 90 and Hepatocellular Carcinoma. International Journal of Clinical Medicine, 11, 43-52.

https://doi.org/10.4236/ijcm.2020.112005

Received: January 17, 2020

Accepted: February 10, 2020

Published: February 13, 2020

Copyright $\odot 2020$ by author(s) and Scientific Research Publishing Inc. This work is licensed under the Creative Commons Attribution International License (CC BY 4.0).

http://creativecommons.org/licenses/by/4.0/

\begin{abstract}
Heat shock protein (HSP) is a kind of protein that mainly acts as a molecular chaperone to participate in the synthesis and folding of proteins, maintain the spatial conformation of proteins and protect cells from damage and other important biological functions. HSP90 plays an important role in maintaining molecular chaperone structure, regulating cell cycle and apoptosis, coordinating hormone signal transduction and promoting wound healing. And HSP90 also plays an important role in the occurrence and progression of tumors. In recent years, HSP90 inhibitors have made some achievements in molecular targeted therapy for malignant tumors, but further research is needed in clinical application. In this paper, the research status of the relationship between hepatocellular carcinoma targeted by heat shock protein 90 was reviewed.
\end{abstract}

\section{Keywords}

Liver Cancer, Heat Shock Protein 90, Molecular Chaperone, Inhibitor, Targeted Therapy

\section{Introduction}

Heat shock protein (HSP), also known as stress protein, is a kind of protein that is synthesized and expressed rapidly after the body is stimulated by external factors (high temperature, hypoxia, cytokine release, etc.) [1]. Heat shock protein (HSP) was first discovered in 1962 by Italian geneticist Ferruccio Ritossa [2] while studying the salivary gland chromosomes of Drosophila larvae. When the ambient temperature increases, the salivary glands of fruit flies bulge, which is called heat shock response (Heat Shock Response, HSR). Heat shock protein (HSP) is a large family of heat stress proteins that exist widely in bacteria, ani${ }^{\star}$ Corresponding author. 
mals and human beings. In vivo, it can mainly play the functions of "molecular chaperone", such as protein folding, transport, transmembrane, conformation stabilization, cell signal transduction, damage protection and so on [3]. According to the relative molecular weight, it can be divided into HSP27, HSP60, HSP40, HSP70, HSP90, HSP110 and so on. Heat shock protein 90 (heat shock protein 90, HSP90) is an important molecular chaperone to maintain the structural stability and function of many kinds of carcinogenic proteins in cells. It is highly expressed in hepatocellular carcinoma, and its substrate protein is a key signal molecule in biological behaviors such as growth and metastasis of hepatocellular carcinoma. HSP90 is closely related to the biological behavior of liver cancer, and it plays an important role in the occurrence, development, metastasis and prognosis of liver cancer [4]. This article reviews the mechanism and expression significance of HSP90 in the occurrence and development of hepatocellular carcinoma, as well as the research of HSP90 inhibitors in hepatocellular carcinoma.

\section{Structure and Function of HSP90}

The HSP90 family is a class of ATP-dependent molecular chaperones with a molecular weight of about $90 \mathrm{kDa}$. HSP90 exists in cells in the form of dimer, and the dimerization of HSP90 is necessary for its intracellular function. Its basic structure consists of three parts: an N-terminal domain (N-domain) that binds to ATP, an intermediate domain (M-domain) that connects the client protein, and a C-terminal domain (C-domain) that connects dimmers [5]. In addition, the HSP90, of eukaryotic cells has a charged region between the $\mathrm{N}$-domain and the M-domain. The N-domain of HSP90 has a mold sequence structure of Bergerat folding, and the binding site region of ATP/ADP is a double-layer $\alpha / \beta$ sandwich structure. There are protein kinase $\mathrm{B}(\mathrm{PKB} / \mathrm{Akt})$ binding sites in the M-domain of HSP90 and the main interaction sites between client proteins. They can also interact with auxiliary molecular chaperones and participate in the hydrolysis of ATP. The main function of M-domain is to bind to substrate peptides such as client proteins or other co-acting molecular chaperones, and its function may be different in the activation process of different types of customer proteins. The C-domain is a necessary region for HSP90 to form a dimer, which contains a highly conserved MEEVD amino acid sequence, which constitutes the core site for the binding of HSP90 to its chaperone tetratricopeptide repeat (TPR). The opening of the C-domain of eukaryotic HSP90 is closely related to the closure of the $\mathrm{N}$-domain. The $\mathrm{C}$ terminal domain also contains a binding site between HSP90 and ATP, but the function of this site is not clear [6] [7].

HSP90 subtypes include cytoplasmic isoforms, HSP90a1/a2 and HSP90 $\beta$, glucose regulated protein 94 (GRP 94) in endoplasmic reticulum and tumor necrosis factor receptor associated protein 1 (TRAP1) in mitochondria [8]. Human HSP90 is divided into two groups according to whether it contains rich glutamine fragments: HSP $90 \alpha$ and HSP90 $\beta$, which are composed of 730 and 724 
amino acids respectively, and their homology is $84 \%$. HSP90 exists mainly as $\alpha$ - $\alpha$ and $\beta$ - $\beta$ homodimers, each homodimer consists of two monomers, and each monomer includes the above three domains. In the non-stress state, the expression of HSP90 accounts for about $1 \% / 2 \%$ of the total protein in the cell, which is thousands of times the average protein content; under the stress condition, the content of HSP90 can be increased to $4 \% / 6 \%$ of the total protein in the cell [9]. The expression of HSP90 $\alpha$ increases after heat induction, which is not necessary in mammals and is related to the maintenance of cell homeostasis under pressure, while HSP90 $\beta$ is sustainable and must exist in mammals, which is related to the life activities of mammals. These proteins are highly homologous in sequence, structure and regulation, and are collectively referred to as HSP90 in this review. As an important molecular chaperone, HSP90 can activate different substrate proteins and then participate in the regulation of a variety of life activities [10].

The main functions of heat shock protein 90 are: 1) molecular chaperone, HSP90 as molecular chaperone, which requires the binding and hydrolysis of ATP, different binding states with ATP/ADP can stabilize the activity of HSP90 substrate protein, promote the ubiquitination of substrate protein and degrade through proteasome pathway, 2) cell cycle regulation, HSP90 can regulate cell division from both positive and negative sides, promote cell division or inhibit cell division, 3) regulation of apoptosis, HSP90 can inhibit the formation of apoptotic bodies, regulate apoptosis, 4) coordinate hormone signal transduction, combine HSP90 with free steroid receptors (estrogen, progesterone, glucocorticoid, etc.) to maintain hormone stability and inactivity, 5) promote chronic refractory wound healing.

\section{Expression of HSP90 in Tumor}

HSP90 is highly expressed in a variety of tumors, which is closely related to the development and prognosis of tumors. It can interfere with tumor proliferation and metastasis by inhibiting HSP90, so it is a great potential antineoplastic drug at present.

HSP90 is highly expressed in breast cancer, which is related to the recurrence and lymph node metastasis of breast cancer. Studies have shown that the high expression of heat shock protein 90 is an independent prognostic factor for breast cancer and is significantly related to the survival time of breast cancer patients [11] [12] [13].

Anti-HSP90 studies have shown that it destroys EGFR-related signaling pathways, down-regulates the activity of HSP90, inhibits tumor cell proliferation, invasion and metastasis, and induces apoptosis of lung cancer cells [14]. HSP90 is highly expressed in lung cancer and promotes the proliferation and metastasis of lung cancer cells. In addition, the high expression of HSP90 in patients with Non small cell lung cancer (NSCLC) is related to shorter overall survival, suggesting that it can be used to predict survival, and the high expression 
of HSP90 can be regarded as an independent prognostic factor of lung cancer [15] [16]. At present, there are a large number of reports about HSP90 inhibitors against lung cancer, but studies have shown that not all lung cancers respond to HSP90 inhibitors [17] [18]. The related mechanism of HSP90 regulating lung cancer at the molecular level still needs to be further explored.

It has been found that thyroid carcinoma (TC) is a tumor with high expression of HSP90. HSP90 is closely related to the occurrence, development and lymph node metastasis of TC [19]. The detection of the expression of HSP90 in TC is helpful to the diagnosis and prognosis of TC, and even can be used as a valuable reference index for differential diagnosis of benign and malignant thyroid lesions and evaluating the prognosis of TC. WHITE et al. [20] through the study of new HSP90 inhibitors, it was found that HSP90 inhibitors inhibit the invasion and metastasis of TC and its cancer stem cell (CSCs). When observing the efficacy of HSP90 inhibitor ganetespib in the treatment of thyroid cancer, Taiwan scholars found that the inhibitor effectively inhibited the proliferation of cancer cells [21].

It was found that berberine (BBR) combined with the second generation HSP90 inhibitor NVP-AUY922 produced a synergistic anti-proliferation effect on colorectal cancer cells sensitive to and insensitive to NVP-AUY922. Dual inhibition of HSP90 may enhance the efficacy of HSP90 inhibitors and overcome the drug resistance of cancer cells. HSP90-ERK-VEGF signaling pathway plays an important role in vascular endothelial cell migration and tubular formation induced by colorectal cancer.

HSP90 is highly [22] expressed in gastric cancer and participates in tumor cell proliferation, differentiation, metastasis and drug resistance. From gastritis to gastric cancer, the expression of HSP90 showed an increasing trend. In gastric cancer, HSP90 is mainly expressed in cytoplasm and a small amount in stromal cells. It is necessary to find the chaperone activity of HSP90 in the progression and invasion of gastric cancer. The high expression of HSP90 in gastric cancer provides an important basis for developing it as a diagnostic marker, therapeutic target and prognostic index [23].

Previous studies have shown that heat shock protein is highly expressed in pancreatic cancer and is closely related to the occurrence, development and metastasis of pancreatic cancer, but it is not a meaningful prognostic indicator of pancreatic cancer. Other studies have shown that HSP90 inhibitors combined with 2,2-difluorodeoxycytidine inhibit the normal growth of pancreatic cancer and completely inhibit tumor liver metastasis. Other studies have shown that HSP90 immunization can play an effective anti-tumor effect. And it can enhance the anti-tumor immunity of the body by stimulating the proliferation of $\mathrm{T}$ lymphocytes.

\section{The Relationship between HSP90 and Hepatocellular Carcinoma}

Hepatocellular carcinoma (HCC) is a common tumor of the digestive system, 
which has a high degree of malignancy and poor prognosis. At present, studies have shown that HCC is a tumor with high expression of HSP90a. The increase of intracellular HSP90 expression is related to the occurrence and development of HCC, and may be an important sign of the development and deterioration of HCC. Using the molecular chaperone function of HSP, inhibiting the high expression of HSP, in HCC by DNA recombinant technique can improve the sensitivity of HCC to radiotherapy and chemotherapy, down-regulate the stability of oncoproteins related to the malignant proliferation of HCC, and then inhibit the proliferation of tumor cells, which is of great significance for the effective prevention and treatment of HCC.

There is a certain correlation between HSP90 $\alpha$ and the biological characteristics of patients with liver cancer. Some studies [24] [25] have shown that the early recurrence and metastasis of patients with liver cancer after treatment are mostly related to the size of tumor focus, satellite focus, vascular tumor thrombus, microvascular invasion, degree of differentiation and pathological classification. It shows that there is a certain relationship between HSP90 $\alpha$ and the prognosis of patients. Studies have shown that [26], the prognosis of patients with HSP90 $\alpha$ negative HCC is significantly better than that of patients with positive HCC (P < 0.05). Li Hui et al. [27] have shown that HSP90 $\alpha$ is related to the metastatic potential of HBV-related HCC, and is expected to be one of the markers for HCC invasion and metastasis, prognosis evaluation and differentiation between HBV-related liver diseases and healthy people.

FU et al. [28] detected the plasma HSP90a protein level in 1652 cases of liver cancer and its control group by ELISA and found that plasma HSP90a could distinguish liver cancer from non-liver cancer patients (sensitivity $92.7 \%$, specificity 91.3\%), plasma HSP90 was more sensitive to distinguish hepatocellular carcinoma than alpha-fetoprotein (93.3\%), and HSP90a could significantly distinguish between AFP negative and AFP localized liver cancer. The plasma HSP90 of patients with liver cancer in surgery and interventional therapy group was higher than that in liver cancer group before treatment. This study shows that HSP90a can be used as a marker for the diagnosis of liver cancer and can also be used to evaluate the therapeutic effect of patients with liver cancer. In 2016, the State Food and Drug Administration approved plasma HSP90 $\alpha$ as a marker for liver cancer, and the kit has been approved for clinical use.

HSP90 targeted cancer therapy begins with the natural inhibitor geldanamycin (GM), which comes from Streptomyces hygroscopicus, showing its anti-proliferation effect by binding to the ATP binding site of HSP90 [29] [30]. However, because of its unstable structure and hepatotoxicity, it cannot be used further [31]. Another important natural inhibitor of HSP90 is free radical (RD), which comes from Bornauden monosporium. It inhibits tumor growth in vitro by attacking the core ATP binding pocket of HSP90, but has been proved to be ineffective in vivo because of its unstable structure [32]. To overcome these initial problems, genetically modified analogues have been developed. Two impor- 
tant transgenic derivatives are 17-AAG (also known as tanespimycin or 17-allylamino-17-demethoxygeldanamycin) and 17-DMAG (also known as alvespimycin or 17-dimethylaminoethylamino-17-demethoxygeldanamycin). The first HSP90 inhibitor evaluated in clinical trials was 17-AAG in 1999 [33]. However, it cannot be used further because of its poor solubility and bioavailability. 17-DMAG shows strong anti-tumor activity and good water solubility, which makes it participate in various phase I clinical trials; however, dose-limiting side effects still exist [34] [35].

Finally, the most advanced and effective HSP90 inhibitors were developed as the second generation derivatives of $\mathrm{RD}$. The first in this category is NVP-AUY922 (also known as luminespib or VER-2296), which shows strong preclinical and clinical efficacy [36] [37]. By far, the most promising second-generation synthetic HSP90 inhibitor based on resorcinol is ganetespib (STA-9090), which binds to the N-terminal ATP binding pocket of HSP90 and destroys the chaperone cycle. The strong anti-tumor activity of this HSP90 inhibitor has been transformed from preclinical success to several clinical studies [38].

AUY922, an inhibitor of heat shock protein 90, blocked the growth of hepatoma cells in vitro and induced apoptosis of hepatoma cells through caspase activation and $\beta$-catenin fragmentation. AUY922 also reduces tumor growth in vivo, indicating that HSP90 has become a promising therapeutic target for HCC. AUY922 inhibits the viability and proliferation of hepatocellular carcinoma cells, but has no effect on normal hepatocytes, and inhibits the activity of HSP90. AUY922 induces apoptosis of hepatocellular carcinoma cells by activating caspases, $\beta$-catenin fragmentation and inhibiting $\beta$-catenin-mediated transcriptional activity [39].

\section{Outlook}

HSP90 is closely related to the biological behavior of liver cancer, and it plays an important role in the occurrence, development, metastasis and prognosis of liver cancer. The increased expression of HSP90 in hepatocellular carcinoma provides an important basis for the development of diagnostic markers, therapeutic targets and prognostic indicators. Extensive research has been carried out on the development of specific HSP90 inhibitors. Many tumor-related signal pathways are blocked by HSP90 inhibitors, which are expected to become potential therapeutic targets to inhibit tumor progression. New HSP90 inhibitors have a stronger inhibitory effect on the growth of liver cancer cells, providing a new way for the treatment of liver cancer. Understanding the function of HSP90 and its molecular mechanism in liver cancer is very important for improving the diagnostic accuracy of liver cancer and developing more effective chemotherapeutic drugs. However, the research and clinical application of HSP90 inhibitors in anti-tumor research and clinical application need to be further developed in the future. 


\section{Conflicts of Interest}

The authors declare no conflicts of interest regarding the publication of this paper.

\section{References}

[1] Liu, H., Dicksved, J., et al. (2014) Heat Shock Proteins: Intestinal Gatekeepers That Are Influenced by Dietary Components and the Gut Microbiota. Pathogens, 1, 187-210. https://doi.org/10.3390/pathogens3010187

[2] Ritossa, F. (1962) A New Puffing Pattern Induced by Temperature Shock and DNP Indrosophila. Experientia, 12, 571-573. https://doi.org/10.1007/BF02172188

[3] Young, J.C., et al. (2001) Hsp90: A Specialized but Essential Protein-Folding Tool. Journal of Cell Biology, 2, 267-273. https://doi.org/10.1083/jcb.200104079

[4] Wu, S.H., Cheng, J. and Zheng, Y.J. (2005) Relationship between Heat Shock Protein Family and Liver Cancer. World Journal of Chinese Digestion, No. 14, 87-92.

[5] Ali, M.M.U., et al. (2006) Crystal Structure of an Hsp90-Nucleotide-p23/Sbal Closed Chaperone Complex. Nature, 7087, 1013-1017. https://doi.org/10.1038/nature04716

[6] Hellenkamp, B., Philipp, W. and Florian, K. (2017) Multidomain Structure and Correlated Dynamics Determined by Self-Consistent FRET Networks. Nature Methods, 14, 174-180. https://doi.org/10.1038/nmeth.4081

[7] Prodromou, C., et al. (1997) Identification and Structural Characterization of the ATP/ADP-Binding Site in the Hsp90 Molecular Chaperone. Cell, 1, 65-75. https://doi.org/10.1016/S0092-8674(00)80314-1

[8] Cheng, G. and Hu, W.X. (2009) Biological Characteristics of Heat Shock Protein 90. Chemistry of Life, 5, 687-690.

[9] Andrija, F. and Pierre, G. (2013) Proteomic Data from Human Cell Cultures Refine Mechanisms of Chaperone-Mediated Protein Homeostasis. Cell Stress Chaperones, 5, 591-605. https://doi.org/10.1007/s12192-013-0413-3

[10] Verba, K.A., et al. (2016) Atomic Structure of Hsp90-Cdc37-Cdk4 Reveals That Hsp90 Traps and Stabilizes an Unfolded Kinase. Science, 6293, 1542-1547. https://doi.org/10.1126/science.aaf5023

[11] Chen, Y., Wang, X. and Cao, C. (2017) Inhibition of HSP90 Sensitizes a Novel Raf/ERK Dual Inhibitor CY-9d in Triple-Negative Breast Cancer Cells. Oncotarget, 61, 104193-104205. https://doi.org/10.18632/oncotarget.22119

[12] Stivarou, T., et al. (2016) Targeting Highly Expressed Extracellular HSP90 in Breast Cancer Stem Cells Inhibits Tumor Growth in Vitro and in Vivo. Cancer Biology \& Therapy, 8, 799-812. https://doi.org/10.1080/15384047.2016.1195041

[13] Lee, C.H., et al. (2012) Inhibition of Heat Shock Protein (Hsp) 27 Potentiates the Suppressive Effect of Hsp90 Inhibitors in Targeting Breast Cancer Stem-Like Cells. Biochimie, 6, 1382-1389. https://doi.org/10.1016/j.biochi.2012.02.034

[14] Rong, B.X. and Yang, S.Y. (2018) Molecular Mechanism and Targeted Therapy of Hsp90 Involved in Lung Cancer: New Discoveries and Developments (Review). International Journal of Oncology, 2, 321-336. https://doi.org/10.3892/ijo.2017.4214

[15] Cedres, S., Felip, E. and Cruz, C. (2018) Activity of HSP90 Inhibiton in a Metastatic Lung Cancer Patient with a Germline BRCA1 Mutation. Journal of the National Cancer Institute, 8, 914-917. https://doi.org/10.1093/jnci/djy012

[16] Sun, Y., Huang, Y.H. and Huang, F.Y. (2018) 3'-epi-12beta-hydroxyfro-side, a New Cardenolide, Induces Cytoprotective Autophagy via Blocking the Hsp90/Akt/mTOR 
Axis in Lung Cancer Cells. Theranostics, 7, 2044-2060. https://doi.org/10.7150/thno.23304

[17] Dong, P.F. and Deng (2017) Clinical Significance of Serum Heat Shock Protein $90 \alpha$ in Patients with Non-Small Cell Lung Cancer. Chinese General Medicine, 19, 2354-2357.

[18] Sun, P.P. and Chen (2017) Research Progress of EGFR Targeted Therapy for Non-Small Cell Lung Cancer. International Respiratory Journal, 2, 148-151.

[19] da Silva, V.C. and Ramos, C.H. (2012) The Network Interaction of the Human Cytosolic $90 \mathrm{kDa}$ Heat Shock Protein Hsp90: A Target for Cancer Therapeutics. Journal of Proteomics, 10, 2790-2802. https://doi.org/10.1016/j.jprot.2011.12.028

[20] White, P.T., et al. (2016) Novel HSP90 Inhibitors Effectively Target Functions of Thyroid Cancer Stem Cell Preventing Migration and Invasion. Surgery, 1, 142-151. https://doi.org/10.1016/j.surg.2015.07.050

[21] Lin, S.F., et al. (2017) Efficacy of an HSP90 Inhibitor, Ganetespib, in Preclinical Thyroid Cancer Models. Oncotarget, 25, 41294-41304.

https://doi.org/10.18632/oncotarget.17180

[22] Su, Y.H., et al. (2015) Targeting of Multiple Oncogenic Signaling Pathways by Hsp90 Inhibitor Alone or in Combination with Berberine for Treatment of Colorectal Cancer. Biochim Biophys Acta, 1853, 2261-2272. https://doi.org/10.1016/j.bbamcr.2015.05.012

[23] Ma, W., et al. (2018) The Progress of Heat Shock Protein 90 in the Study of Gastric Cancer. Medical Science Journals of Central South China, 1, 98-112.

[24] Cheng, Z.J., et al. (2015) Risk Factors and Management for Early and Late Intrahepatic Recurrence of Solitary Hepatocellular Carcinoma after Curative Resection. $H P B, 5,422-427$. https://doi.org/10.1111/hpb.12367

[25] Sohn, W., et al. (2014) HBV DNA and HBsAg Levels as Risk Predictors of Early and Late Recurrence after Curative Resection of HBV-Related Hepatocellular Carcinoma. Annals of Surgical Oncology, 7, 2429-2435. https://doi.org/10.1245/s10434-014-3621-x

[26] Sun, S.P., et al. (2003) Sun Level, the Expression and Significance of HSP90 $\alpha$ in Hepatocellular Carcinoma. Chinese Journal of Basic and Clinical General Surgery, No. 3, 243-245.

[27] Li, H., et al. (2014) The Significance of Heat Shock Protein 90 a Expression in HBV-Related Hepatocellular Carcinoma. Journal of Guangzhou Medical University, 42, 21-24.

[28] Fu, Y., et al. (2017) Plasma Heat Shock Protein 90alpha as a Biomarker for the Diagnosis of Liver Cancer: An Official, Large-Scale, and Multicenter Clinical Trial. EBioMedicine, 24, 56-63. https://doi.org/10.1016/j.ebiom.2017.09.007

[29] Ochiana, S.O., Taldone, T. and Chiosis, G. (2014) Designing Drugs against Hsp90 for Cancer Therapy. In: Houry, W.A., Ed., the Molecular Chaperones Interaction Networks in Protein Folding and Degradation, Interactomics and Systems Biology, Vol. 1, Springer, Berlin, 151-183. https://doi.org/10.1007/978-1-4939-1130-1 7

[30] Patel, H.J., et al. (2011) Advances in the Discovery and Development of Heat-Shock Protein 90 Inhibitors for Cancer Treatment. Expert Opinion on Drug Discovery, 6, 559-587. https://doi.org/10.1517/17460441.2011.563296

[31] Supko, J.G., et al. (1995) Preclinical Pharmacologic Evaluation of Geldanamycin as an Antitumor Agent. Cancer Chemotherapy and Pharmacology, 36, 305-315. https://doi.org/10.1007/BF00689048 
[32] Soga, S., et al. (2003) Development of Radicicol Analogues. Current Cancer Drug Targets, 3, 359-369. https://doi.org/10.2174/1568009033481859

[33] Banerji, U., et al. (2005) Phase I Pharmacokinetic and Pharmacodynamic Study of 17-Allylamino 17-Demethoxygeldanamycin in Patients with Advanced Malignancies. Journal of Clinical Oncology, 23, 4152-4161. https://doi.org/10.1200/JCO.2005.00.612

[34] Lancet, J.E., et al. (2010) Phase I Study of the Heat Shock Protein 90 Inhibitor Alvespimycin (KOS-1022, 17-DMAG) Administered Intravenously Twice Weekly to Patients with Acute Myeloid Leukemia. Leukemia, 24, 699-705. https://doi.org/10.1038/leu.2009.292

[35] Kummar, S., et al. (2010) Phase I Trial of 17-Dimethylaminoethylamino-17Demethoxygeldanamycin (17-D.M.A.G.), a Heat Shock Protein Inhibitor, Administered Twice Weekly in Patients with Advanced Malignancies. European Journal of Cancer, 46, 340-347. https://doi.org/10.1016/j.ejca.2009.10.026

[36] Brough, P.A., et al. (2008) 4,5-Diarylisoxazole Hsp90 Chaperone Inhibitors: Potential Therapeutic Agents for the Treatment of Cancer. Journal of Medicinal Chemistry, 51, 196-218. https://doi.org/10.1021/jm701018h

[37] Shiotsu, Y., et al. (2000) Novel Oxime Derivatives of Radicicol Induce Erythroid Differentiation Associated with Preferential G(1) Phase Accumulation against Chronic Myelogenous Leukemia Cells through Destabilization of Bcr-Abl with Hsp90 Complex. Blood, 96, 2284-2291. https://doi.org/10.1182/blood.V96.6.2284

[38] Chatterjee, S. and Burns, T.F. (2017) Targeting Heat Shock Proteins in Cancer: A Promising Therapeutic Approach. International Journal of Molecular Sciences, 18, 1978. https://doi.org/10.3390/ijms18091978

[39] Augello, G., et al. (2019) Targeting HSP90 with the Small Molecule Inhibitor AUY922 (Luminespib) as a Treatment Strategy against Hepatocellular Carcinoma. International Journal of Cancer, 144, 2613-2624. https://doi.org/10.1002/ijc.31963 


\section{Abbreviation Note List}

HCC: Hepatocarcinoma

HSP: Heat shock protein

HSR: Heat shock response

$\mathrm{N}$-domain: N-terminal domain

M-domain: Intermediate domain

C-domain: C-terminal domain

PKB/Akt: Protein kinaseB

TPR: Tetratricopeptide repeat

Grp94: Glucose regulated protein 94

TRAP1: Tumor necrosis factor receptor associated protein 1

TC: Thyroid Cancer

NSCLC: Non small cell lung cancer

CSC: Cancer stem cell

BBR: Berberine

GM: Geldanamycin

RD: Radicicol

17-AAG: Tanespimycin

17-DMAG: Alvespimycin

NVP-AUY922: Luminespib

STA-9090: Ganetespib 